\title{
Model Atmosphere Spectrum Fit to the Soft X-Ray Outburst Spectrum of SS Cyg
}

\author{
V. F. Suleimanov ${ }^{1,2}$, C. W. Mauche ${ }^{3}$, R. Ya. Zhuchkov ${ }^{2}$, K. Werner ${ }^{1}$ \\ ${ }^{1}$ Institute for Astronomy and Astrophysics, Kepler Center for Astro and Particle Physics, Eberhard Karls University, \\ Sand 1, 72076 Tübingen, Germany \\ ${ }^{2}$ Kazan (Volga region) Federal University, Kremlevskaya str. 18, 42008 Kazan, Russia \\ ${ }^{3}$ Lawrence Livermore National Laboratory, L-473, 7000 East Ave., Livermore, CA 94550, USA \\ Corresponding author: suleimanov@astro.uni-tuebingen.de
}

\begin{abstract}
The X-ray spectrum of SS Cyg in outburst has a very soft component that can be interpreted as the fast-rotating optically thick boundary layer on the white dwarf surface. This component was carefully investigated by Mauche (2004) using the Chandra LETG spectrum of this object in outburst. The spectrum shows broad $(\approx 5 \AA)$ spectral features that have been interpreted as a large number of absorption lines on a blackbody continuum with a temperature of $\approx 250 \mathrm{kK}$. Because the spectrum resembles the photospheric spectra of super-soft X-ray sources, we tried to fit it with high gravity hot LTE stellar model atmospheres with solar chemical composition, specially computed for this purpose. We obtained a reasonably good fit to the 60-125 ̊̊ spectrum with the following parameters: $T_{\text {eff }}=190 \mathrm{kK}, \log g=6.2$, and $N_{\mathrm{H}}=8 \cdot 10^{19} \mathrm{~cm}^{-2}$, although at shorter wavelengths the observed spectrum has a much higher flux. The reasons for this are discussed. The hypothesis of a fast rotating boundary layer is supported by the derived low surface gravity.
\end{abstract}

Keywords: cataclysmic variables - dwarf novae - radiation transfer - X-rays - individual: $\mathrm{SS}$ Cyg $\equiv \mathrm{BD}+42^{\circ} 4189 \mathrm{a}$.

\section{Introduction}

SS Cyg is one of the brightest cataclysmic variables (CVs), one of the best-studied dwarf nova stars (Warner 1995), and was the first CV discovered in X-ray radiation (Rappaport et al. 1974). The properties of the $\mathrm{X}$-ray radiation of this close binary system have been extensively investigated, and are observed to be dramatically different in quiescence and in outburst. In quiescence, the $\mathrm{X}$-ray spectrum is hard and can be described by an optically thin hot $(k T \approx 20 \mathrm{keV})$ plasma with an observed flux $\approx 2 \cdot 10^{-10} \mathrm{erg} \mathrm{s}^{-1} \mathrm{~cm}^{-2}$. In outburst, this hard component decreases by a factor of ten, the plasma temperature is reduced to $\sim 6-8 \mathrm{keV}$, and an additional soft component appears with a blackbody temperature $\approx 200-300 \mathrm{kK}$ (Córdova et al. 1980; McGowan et al. 2004; Ishida et al. 2009). It is commonly accepted that the $\mathrm{X}$-ray radiation of non-magnetic CVs arises in the boundary layer (BL) between the white dwarf (WD) and the accretion disc (Pringle \& Savonije 1979; Tylenda 1981; Patterson \& Raymond 1985a, b; Kley 1991), which are optically thick at high accretion rates $\left(\dot{M}>10^{16} \mathrm{~g} \mathrm{~s}^{-1}\right)$ and optically thin at lower accretion rates.

The soft X-ray spectrum of SS Cyg in outburst was carefully investigated by Mauche (2004) using a high- resolution spectrum obtained with the Chandra LETG. He phenomenologically described the observed 40-130 $\AA$ spectrum by a blackbody with temperature $T \approx 250$ $\mathrm{kK}$ and numerous broad absorption features of ions of cosmically abundant $\mathrm{O}, \mathrm{Ne}, \mathrm{Mg}, \mathrm{Si}, \mathrm{S}$, and Fe. The BL luminosity and WD spin were also evaluated in this work. On the other hand, this spectrum looks like the photospheric spectra of super-soft X-ray sources (Lanz et al. 2005; Rauch et al. 2010; van Rossum 2012), so it probably could be described using the spectra of hot stellar model atmospheres. Boundary layers possibly rotate with almost Keplerian velocities and could have reduced (in comparison with WD) surface gravities close to the local Eddington limit. Therefore, we consider close to Eddington limit models in the present work. Here we present our attempt to fit the Chandra LETG spectrum of SS Cyg using such model spectra. We also make estimates of the BL parameters in the context of our model fits.

\section{Model Atmospheres}

The version of the LTE computer code ATLAS (Kurucz 1970; 1993), modified by us to deal with high temperatures (Ibragimov et al. 2003; Suleimanov \& Werner 2007), was used to model high temperature at- 
mospheres. In this code, local thermodynamic equilibrium (LTE) is assumed and the pressure ionization effects using the occupation probability formalism (Hummer \& Mihalas 1988) as described by Hubeny et al. (1994) are taken into account. Coherent electron scattering together with the free-free and bound-free transitions of all ions of the 15 most abundant elements using cross-sections from Verner \& Yakovlev (1995) were adopted for the continuum opacity. Line blanketing is also included using $\sim 25000$ spectral lines from the CHIANTI, Version 3.0, atomic database (Dere et al. 1997).
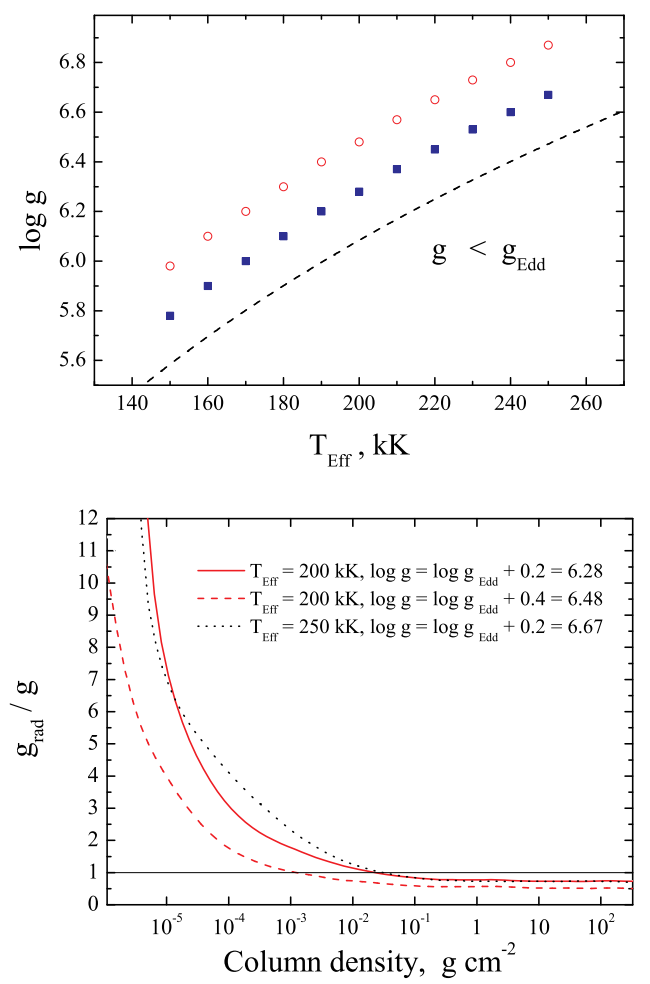

Figure 1: Top panel: Positions of the computed model atmospheres in the $T_{\text {eff }}-\log g$ plane. The dashed curve demarcates the Eddington $\operatorname{limit}\left(\log g=\log g_{\text {Edd }}\right)$. Bottom panel: The relative radiation force vs. depth for various model atmospheres.

Twenty-two model atmospheres with solar chemical composition were computed using the described code. The effective temperatures of the models range between $150 \mathrm{kK}$ and $250 \mathrm{kK}$ with a step of $10 \mathrm{kK}$. Two values of the surface gravity for each effective temperature, namely $\log g=\log g_{\text {Edd }}+0.2$ and $\log g=\log g_{\text {Edd }}+0.4$, were used. Here $\log g_{\mathrm{Edd}}=\log \left(\sigma_{\mathrm{e}} \sigma_{\mathrm{SB}} T_{\mathrm{eff}}^{4} / c\right)=4.88+$ $4 \log \left(T_{\text {eff }} / 10^{5} K\right)$ is the surface gravity that has an equal radiation pressure force for a given $T_{\text {eff }}$, and $\sigma_{\mathrm{e}} \approx 0.34$ $\mathrm{cm}^{2} \mathrm{~g}^{-1}$ is the electron scattering opacity for the assumed solar chemical composition. The positions of the computed models on the $T_{\text {eff }}-\log g$ plane are shown in Fig. 1 (top panel). For the considered model atmospheres, a radiation pressure force $g_{\mathrm{rad}}$ due to spectral lines becomes larger than the surface gravity at the upper atmospheric layers (see Fig. 1, bottom panel). To enforce hydrostatic equilibrium, we took a gas pressure equal to $10 \%$ of the total pressure $\left(P_{\text {gas }}=0.1 P_{\text {tot }}\right)$ at all atmospheric layers where $g_{\mathrm{rad}}>g$.

As shown in Fig. 2, the computed emergent spectra are dominated by a forest of absorption lines, and have to be convolved with the LETG spectral resolution and the interstellar gas transmission to be compared with the observed spectrum of SS Cyg (see Fig. 3). The spectra of models with different surface gravities are sufficiently different to discriminate them from a comparison with the observed spectrum (see Fig. 2, bottom panel).

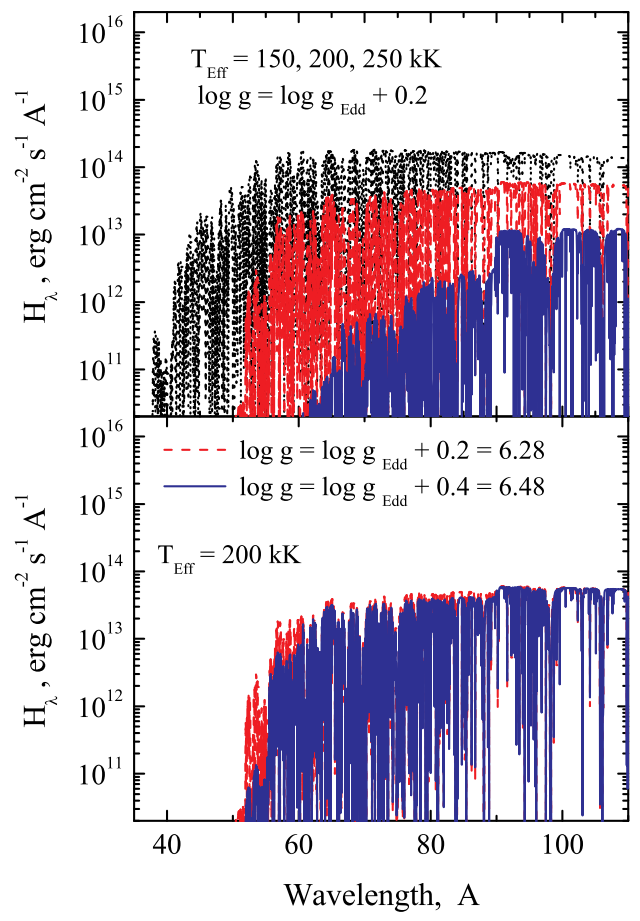

Figure 2: Top Panel: Emergent spectra of three model atmospheres with the same $\log g=\log g_{\text {Edd }}+0.2$ and various effective temperatures: $150 \mathrm{kK}$ (solid curves), $200 \mathrm{kK}$ (dashed curves), and $250 \mathrm{kK}$ (dotted curves). Bottom panel: Emergent spectra of two model atmospheres with the same effective temperature $(200 \mathrm{kK})$ and different $\log g$.

\section{Results}

The model spectra convolved with the Chandra LETG spectral resolution $\Delta \lambda=0.05 \AA$ were used to fit the observed soft X-ray spectrum of SS Cyg. The interstellar absorption (with the hydrogen column number den- 
sity $N_{H}$ as a fitting parameter) was also taken into account. The observed spectrum was fitted in the 60-125 $\AA$ wavelength range because at the shorter wavelengths our model spectra could be incorrect (see next section). The best-fit model parameters are $T_{\text {eff }}=190 \mathrm{kK}$, $N_{\mathrm{H}}=8 \cdot 10^{19} \mathrm{~cm}^{-2}$, and normalization $K=7.82 \cdot 10^{-26}$, and correspond to the models with the lower surface gravity $\log g=\log g_{\text {Edd }}+0.2$. The reduced $\chi^{2}=3.9$ is relatively large, hence the formal parameter errors are large, too, and we have not attempted to determine them. The best-fit model spectrum together with the observed spectrum is shown in Fig. 3. The contours of $\chi^{2}$ on the $T_{\text {eff }}-\log N_{\mathrm{H}}$ parameter plane are shown in Fig. 4. The normalization can be expressed as $K=f R_{\mathrm{WD}}^{2} / d^{2}$ where $d$ is the distance to SS Cyg and $f$ is the WD fractional area occupied by the BL, which can be expressed as the relative BL extension along the WD surface $f \approx\left(2 \pi R_{\mathrm{WD}} 2 H_{\mathrm{BL}}\right) /\left(4 \pi R_{\mathrm{WD}}^{2}\right)=H_{\mathrm{BL}} / R_{\mathrm{WD}}$.

The basic properties of the BL can be derived from the obtained fit parameters. Using the same system and WD parameters as used by Mauche (2004) - $M_{\mathrm{WD}}=1 M_{\odot}, R_{\mathrm{WD}}=5.5 \cdot 10^{8} \mathrm{~cm}$ (therefore, model $\log g=8.46), d=160 \mathrm{pc}$, and an accretion disk bolometric luminosity in outburst $L_{\text {Disk }}=10^{35}$ erg $\mathrm{s}^{-1}$ - we obtain the fractional area of the BL $f=6.3 \cdot 10^{-2}\left(5 \cdot 10^{-3}\right)$, the bolometric BL luminosity $L_{\mathrm{BL}}=1.8 \cdot 10^{34}\left(5 \cdot 10^{33}\right) \mathrm{erg} \mathrm{s}^{-1}$, and the relative $\mathrm{BL}$ luminosity $L_{\mathrm{BL}} / L_{\mathrm{Disk}}=0.18(0.05)$, where the best-fit parameter values obtained by Mauche (2004) are shown in parentheses for comparison.

The spin period of the WD in SS Cyg is 12 (9) s as inferred using the relation between the BL and accretion disk luminosities (Kluźniak 1987; Kley1991)

$$
L_{\mathrm{BL}} / L_{\mathrm{Disk}}=\left[1-\Omega_{\mathrm{WD}} / \Omega_{\mathrm{K}}\left(R_{\mathrm{WD}}\right)\right]^{2},
$$

where $\Omega_{\mathrm{K}}\left(R_{\mathrm{WD}}\right)$ is the Kepler angular velocity at the $\mathrm{WD}$ radius.

The accepted model gravity on the WD surface in SS Cyg $\left(\log g_{\mathrm{WD}}=8.46\right)$ is more than two orders of magnitude higher than the obtained BL effective surface gravity $\log g_{\text {eff }}=6.2$. The surface gravity of the $\mathrm{BL}$ can be reduced only by fast rotation of the accreting matter

$$
\begin{aligned}
g_{\mathrm{eff}} & =g_{\mathrm{WD}}-\Omega_{\mathrm{BL}}^{2} R_{\mathrm{WD}} \\
& =g_{\mathrm{WD}}\left(1-\left[\Omega_{\mathrm{BL}} / \Omega_{\mathrm{K}}\left(R_{\mathrm{WD}}\right)\right]^{2}\right)
\end{aligned}
$$

Therefore, a relative $\mathrm{BL}$ angular velocity $\Omega_{\mathrm{BL}} / \Omega_{\mathrm{K}}\left(R_{\mathrm{WD}}\right) \approx 0.98$ was obtained using this relation.

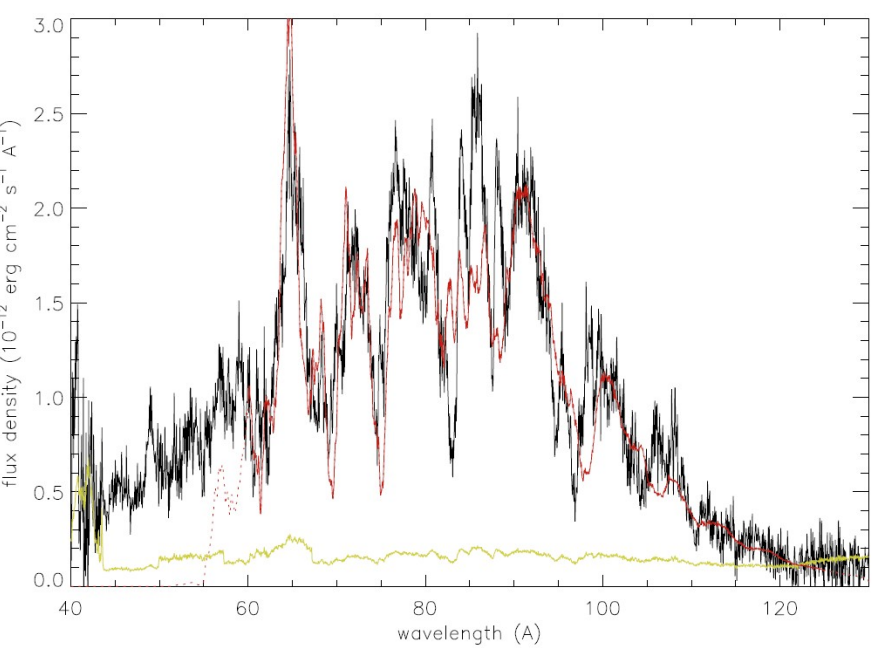

Figure 3: The Chandra LETG spectrum of SS Cyg in outburst (thick black curve) and the best-fit model atmosphere spectrum with $T_{\text {eff }}=190 \mathrm{kK}, \log g=6.2$, and $\log N_{\mathrm{H}}=19.9$ (thin red curve). The fitting was performed in the 60-125 $\AA$ wavelength range. The model spectrum at shorter wavelengths is shown by the dashed red curve.

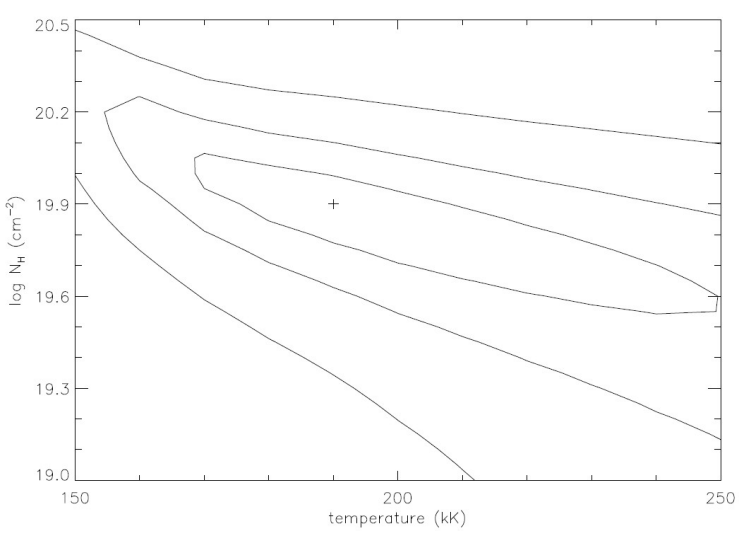

Figure 4: Position of the best-fit model in the $T_{\text {eff }}-\log N_{\mathrm{H}}$ parameter plane and contours of $\chi^{2}=$ $[1.5,3,6] \chi_{\min }^{2}$.

\section{Discussion and Conclusion}

We present here the results of fitting the SS Cyg Chandra LETG spectrum in outburst with model atmosphere spectra. The obtained best fit model spectrum does not describe the observed spectrum at short wavelengths $(<60 \AA)$ and in the $82-90 \AA$ wavelength region and, therefore, it is not statistically acceptable $\left(\chi^{2} /\right.$ dof $\left.=3.9\right)$. These deficiencies can be connected with model shortcomings: the most important ignored effect is atmosphere expansion due to a spectral linedriven stellar wind. This expansion can be significant 
because $g_{\mathrm{rad}}>g$ at the outer layers of our model atmospheres (see also van Rossum 2012). In addition, non-LTE effects could be important (see, e.g., Rauch et al. 2010), the chemical composition may differ from solar, and, finally, the atomic data are almost certainly neither complete nor entirely accurate.

The second important shortcoming is connected with a likely complicated BL structure with a distribution of effective temperatures and surface gravities over its surface. Therefore, a simple one-zone BL model presented here is most probably insufficient and more sophisticated BL models have to be considered.

Nevertheless, the spectral modeling presented here supports a BL in SS Cyg that is, to first approximation, a hot $(\approx 190 \mathrm{kK})$, fast rotating $\left[\Omega_{\mathrm{BL}} \approx 0.98 \Omega_{\mathrm{K}}\left(R_{\mathrm{WD}}\right)\right]$, narrow $\left(H_{\mathrm{BL}} \approx 0.063 R_{\mathrm{WD}}\right)$ belt on the WD surface.

\section{Acknowledgement}

This work is supported by the DFG SFB / Transregio 7 "Gravitational Wave Astronomy" (V.S.) and the Russian Foundation for Basic Research (grant 12-02-97006r-povolzhe-a) (R.Zh.). C.W.M.'s contribution to this work was performed under the auspices of the U.S. Department of Energy by Lawrence Livermore National Laboratory under Contract DE-AC52-07NA27344.

\section{References}

[1] Córdova, F. A., Chester, T. J., Tuohy, I. R., \& Garmire, G. P. 1980, ApJ, 235, 163

[2] Dere, K. P., Landi, E., Mason, H. E., Monsignori Fossi, B. C., \& Young, P. R. 1997, A\&ASS, 125, 149

[3] Hubeny, I., Hummer, D. G., \& Lanz, T. 1994, A\&A, 282, 151

[4] Hummer, D. G., \& Mihalas, D. 1988, ApJ, 331, 794 doi: $10.1086 / 166600$

[5] Ibragimov, A. A., Suleimanov, V. F., Vikhlinin, A., \& Sakhibullin, N. A. 2003, Astronomy Reports, 47, 186 doi:10.1134/1.1562213

[6] Ishida, M., Okada, S., Hayashi, T., Nakamura, R., Terada, Y., Mukai, K., \& Hamaguchi, K. 2009, PASJ, 61,77

[7] Kley, W. 1991, A\&A, 247, 95

[8] Kluźniak, W. 1987, Ph.D. thesis, Stanford Univ.

[9] Kurucz, R. L. 1970, SAO Special Report, 309

[10] Mauche, C. W. 2004, ApJ, 610, 422
[11] McGowan, K. E., Priedhorsky, W. C., \& Trudolyubov, S. P. 2004, ApJ, 601, 1100 doi:10.1086/380758

[12] Patterson, J., \& Raymond, J. C. 1985a, ApJ, 292, 535 doi:10.1086/163187

[13] Patterson, J., \& Raymond, J. C. 1985b, ApJ, 292, 550 doi:10.1086/163188

[14] Pringle, J. E., \& Savonije, G. J. 1979, MNRAS, 187, 777 doi:10.1093/mnras/187.4.777

[15] Rauch, T., Orio, M., Gonzales-Riestra, R., Nelson, T., Still, M., Werner, K., \& Wilms, J. 2010, ApJ, 717, 363 doi:10.1088/0004-637X/717/1/363

[16] Rappaport, S., Cash, W., Doxsey, R., McClintock, J., \& Moore, G. 1974, ApJ, 187, L5 doi:10.1086/181378

[17] Suleimanov, V., \& Werner, K. 2007, A\&A, 466, 661

[18] Tylenda, R. 1981, Acta Astr., 31, 267

[19] van Rossum, D. R. 2012, ApJ, 756, 43

[20] Verner, D. A., \& Yakovlev, D. G. 1995, A\&ASS, 109, 125

[21] Warner, B. 1995, Cambridge Astrophysics Series, 28

\section{DISCUSSION}

DMITRY KONONOV: What is a reason to initially use the solar chemical composition instead of chemical compositions for stars of later spectral type?

VALERY SULEIMANOV: Unfortunately, we do not know the chemical composition of the secondary in SS Cyg. Therefore, we started from the most conservative case, which is the solar chemical composition.

JAN-UWE NESS: The assumed WD mass in the

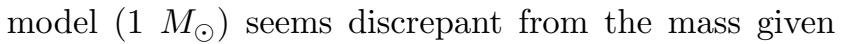
in the introduction $\left(0.55 M_{\odot}\right)$, a factor 2 . This suggest that all parameters of the model may be discrepant from reality, so what do we learn?

VALERY SULEIMANOV: Sorry, you mixed up the white dwarf mass with the mass of the secondary ( 0.55 $\left.M_{\odot}\right)$.

DMITRY BISIKALO: How can you discriminate between a boundary layer and a pseudo-photosphere or a hot disk halo? 
VALERY SULEIMANOV: The observed soft X- tive temperature $(\approx 190 \mathrm{kK})$ would have a radius that ray luminosity is a discrimination factor. Any pseudophotosphere or an optically thick hot disk halo with this low surface gravity $(\log g \approx 6.2)$ and the derived effecis tens of times larger than the white dwarf radius, and the luminosity would be hundreds of times larger than the observed luminosity. 